\title{
State of the art in abdominal MRI structured reporting: a review
}

\author{
Arnaldo Stanzione ${ }^{1} \cdot$ Francesca Boccadifuoco $^{1} \cdot$ Renato Cuocolo $^{2}\left(\mathbb{0} \cdot\right.$ Valeria Romeo $^{1} \cdot$ Pier Paolo Mainenti $^{3}$. \\ Arturo Brunetti ${ }^{1}$. Simone Maurea ${ }^{1}$
}

Received: 4 August 2020 / Revised: 27 August 2020 / Accepted: 3 September 2020 / Published online: 16 September 2020

(c) The Author(s) 2020

\begin{abstract}
In the management of several abdominal disorders, magnetic resonance imaging (MRI) has the potential to significantly improve patient's outcome due to its diagnostic accuracy leading to more appropriate treatment choice. However, its clinical value heavily relies on the quality and quantity of diagnostic information that radiologists manage to convey through their reports. To solve issues such as ambiguity and lack of comprehensiveness that can occur with conventional narrative reports, the adoption of structured reporting has been proposed. Using a checklist and standardized lexicon, structured reports are designed to increase clarity while assuring that all key imaging findings related to a specific disorder are included. Unfortunately, structured reports have their limitations too, such as risk of undue report simplification and poor template plasticity. Their adoption is also far from widespread, and probably the ideal balance between radiologist autonomy and report consistency of has yet to be found. In this article, we aimed to provide an overview of structured reporting proposals for abdominal MRI and of works assessing its value in comparison to conventional free-text reporting. While for several abdominal disorders there are structured templates that have been endorsed by scientific societies and their adoption might be beneficial, stronger evidence confirming their imperativeness and added value in terms of clinical practice is needed, especially regarding the improvement of patient outcome.
\end{abstract}

Keywords Structured report $\cdot$ Abdominal MRI $\cdot$ Evidence-based medicine $\cdot$ Report quality $\cdot$ Standardized lexicon

\begin{tabular}{ll}
\multicolumn{2}{l}{ Abbreviations } \\
MRI & Magnetic resonance imaging \\
NR & Narrative report \\
SR & Structured report \\
HCC & Hepatocellular carcinoma (HCC) \\
LI-RADS & Liver Imaging-Reporting and Data System \\
PI-RADS & Prostate Imaging-Reporting and Data System \\
O-RADS & Ovarian-Adnexal Imaging-Reporting and \\
& Data System \\
VI-RADS & Vesical Imaging-Reporting and Data System \\
MRE & Magnetic resonance enterography \\
SAR & Society of Abdominal Radiology
\end{tabular}

Renato Cuocolo

renato.cuocolo@unina.it

1 Department of Advanced Biomedical Sciences, University of Naples "Federico II", Naples, Italy

2 Department of Clinical Medicine and Surgery, University of Naples "Federico II", Via S. Pansini, 5, 80131 Naples, Italy

3 Institute of Biostructures and Bioimaging, National Research Council, Naples, Italy
AGA American Gastroenterological Association

FIA Fistula-in-ano

\section{Background}

Allowing to evaluate both morphological and functional features of oncologic and non-oncologic diseases, magnetic resonance imaging (MRI) plays a major role in abdominal imaging. While a proper acquisition protocol and the correct identification and interpretation of key imaging findings are of uttermost importance to achieve optimal diagnostic accuracy, the quality of MRI reports should be considered equally crucial. Indeed, sentences that convey diagnostic uncertainty are frequently present in MRI reports, which are also often lacking in terms of clarity and extensiveness, thus leading to potential misunderstandings with the referring physicians $[1,2]$. Therefore, the transition from narrative (NR) to structured reports (SR) has been considered a valid strategy to increase the value of diagnostic imaging [3]. The conventional NR are based on free-text and personalized language, characteristics that allow flexibility in reporting 
at the expense of variability in length, lexicon, style, and content $[4,5]$. On the other hand, the main features of SR are format, content, and language standardization. More specifically, SR are organized into paragraphs with appropriate headings to identify essential sections (e.g., clinical setting and indication, imaging protocol, radiological findings, conclusions). Additionally, key imaging findings are arranged in a check-list style, which is contextualized and adapted based on the specific disorder/clinical indication, so that they are easily identifiable and cannot be mistakenly left out by the reporting radiologist. Finally, a standardized language must be embraced in SR, as it allows to minimize misinterpretations of pathological and normal findings and facilitate comparability of reports $[2,3,6]$.

There are several studies suggesting that referring physicians prefer SR over NR as well as that SRs offer many advantages (e.g., fewer diagnostic error, higher consistency and comprehensiveness) leading to an overall improvement of report quality $[2,7,8]$. To promote the adoption of SR, the Radiological Society of North America offers a free and multilanguage radiology reporting template library (www. radreport.org). Similarly, the American College of Radiology provides a standardized framework for reporting on imaging findings (www.acr.org/Clinical-Resources/Repor ting-and-Data-Systems). However, while scoring systems enhance MRI reporting through standardization, these can be used in NR as well as in SR and the concepts of standardized and structured reporting appear separate and complementary rather than redundant, with SR covering the creation of the report and its layout [9]. Furthermore, the transition from NR to SR is progressing slowly, and the use of SR is prevalent within subspecialty groups rather than widespread [10]. Finally, there are still radiologists who prefer NR as they are more accustomed to it, while others believe that SR could be limiting compared to their skill in fluent speech, diverting their focus from the images and reducing their efficiency $[2,8]$.

With this review we aimed to identify the main current applications of SR in abdominal MRI and discuss relevant evidence available in the scientific literature regarding its comparison to NR.

\section{Current applications}

An overview of abdominal diseases for which structured MRI reporting recommendations released by scientific societies are presently available in the literature can be found in Table 1. Most of these recommendations are focused on oncologic disorders (e.g., renal masses, prostate cancer, endometrial cancer) and were drafted to meet a specific diagnostic need (e.g., lesion detection, staging). However, there are also some diseases for which SR templates have been proposed by independent working groups and experts (e.g., perianal fistulas). To shed light on the actual impact that SR widespread adoption might have in clinical practice, the major results of studies performing a direct comparison between SR and NR are presented. The main characteristics of these are reported in Table 2. In the following paragraphs, organized by district and pathology, a more extensive overview is proposed.

\section{Upper abdomen}

\section{Hepatocellular carcinoma}

Hepatocellular carcinoma (HCC) is the most common primary liver malignancy and a leading cause of cancerrelated deaths [11]. It can be diagnosed noninvasively using

Table 1 List of diseases with recommendations for structured abdominal MRI reporting from scientific societies

\begin{tabular}{|c|c|c|c|c|}
\hline District & Disease & Purpose & Society & Resources \\
\hline \multirow[t]{2}{*}{ Upper abdomen } & Hepatocellular carcinoma & Detection & ACR & {$[15]$} \\
\hline & Renal masses & Characterization & SAR & {$[25]$} \\
\hline \multirow[t]{5}{*}{ Lower abdomen } & Prostate cancer & Detection & ACR, ESUR & {$[15]$} \\
\hline & Bladder cancer & Staging & EAU, JSAR & {$[35]$} \\
\hline & Endometrial cancer $^{\mathrm{a}}$ & Staging & ESUR & {$[48]$} \\
\hline & $\begin{array}{l}\text { Female genital tract congenital } \\
\text { anomalies }\end{array}$ & Detection & ESUR & {$[52]$} \\
\hline & Endometriosis & Detection and disease assessment & ESUR & {$[55]$} \\
\hline \multirow[t]{2}{*}{ Gastrointestinal tract } & Rectal cancer & Staging & ESGAR & {$[64]$} \\
\hline & Crohn's disease & Detection and disease assessment & SAR, AGA & [70] \\
\hline
\end{tabular}

$A C R$ American College of Radiology, SAR Society of Abdominal Radiology, ESUR European Society of Urogenital Radiology, EAU European Association of Urology, JSAR Japanese Society of Abdominal Radiology, ESGAR European Society of Gastrointestinal and Abdominal Radiology, AGA American Gastroenterological Association

${ }^{\text {a }}$ Structured reporting template proposed by the Society of Abdominal Radiology available at radreport.org 


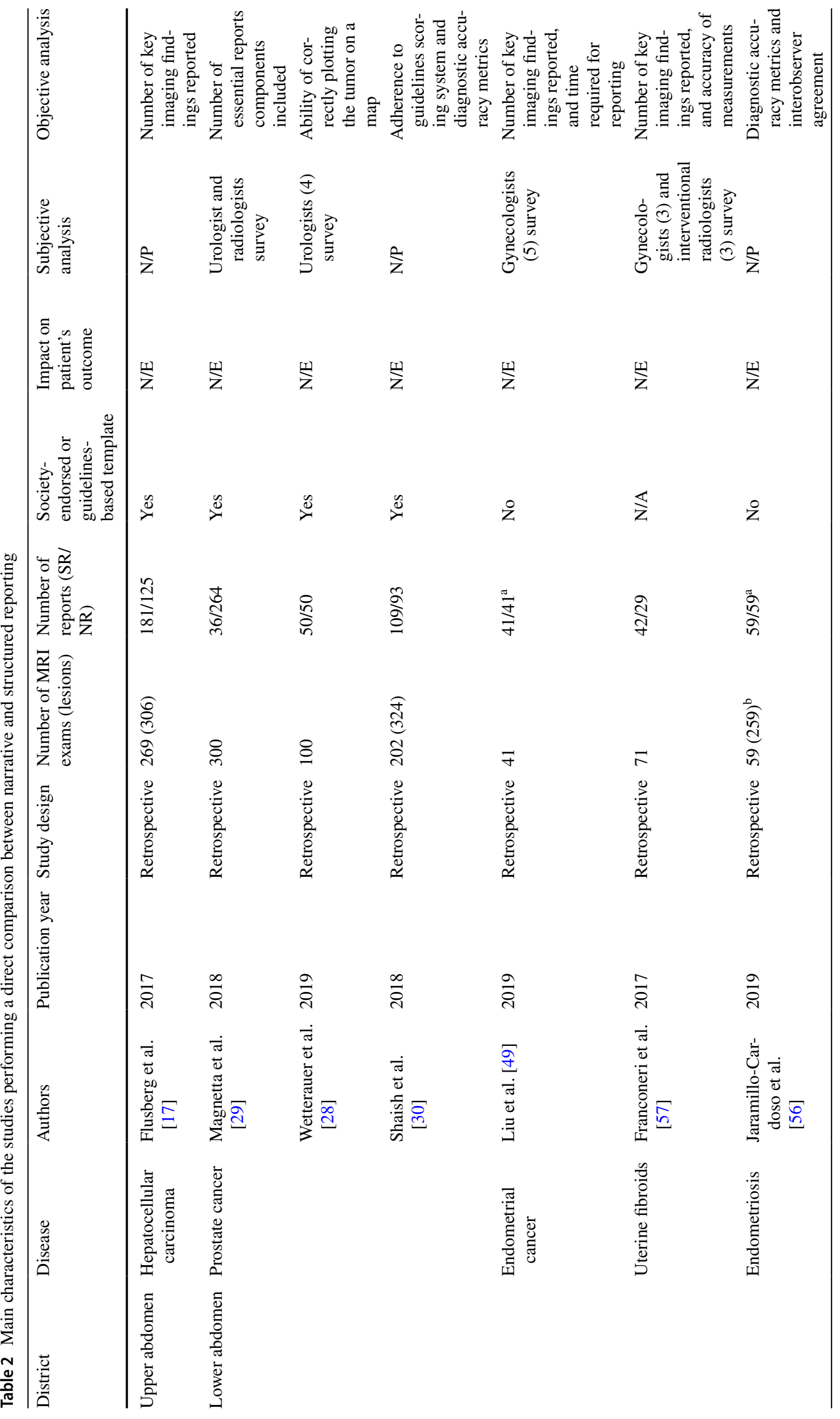




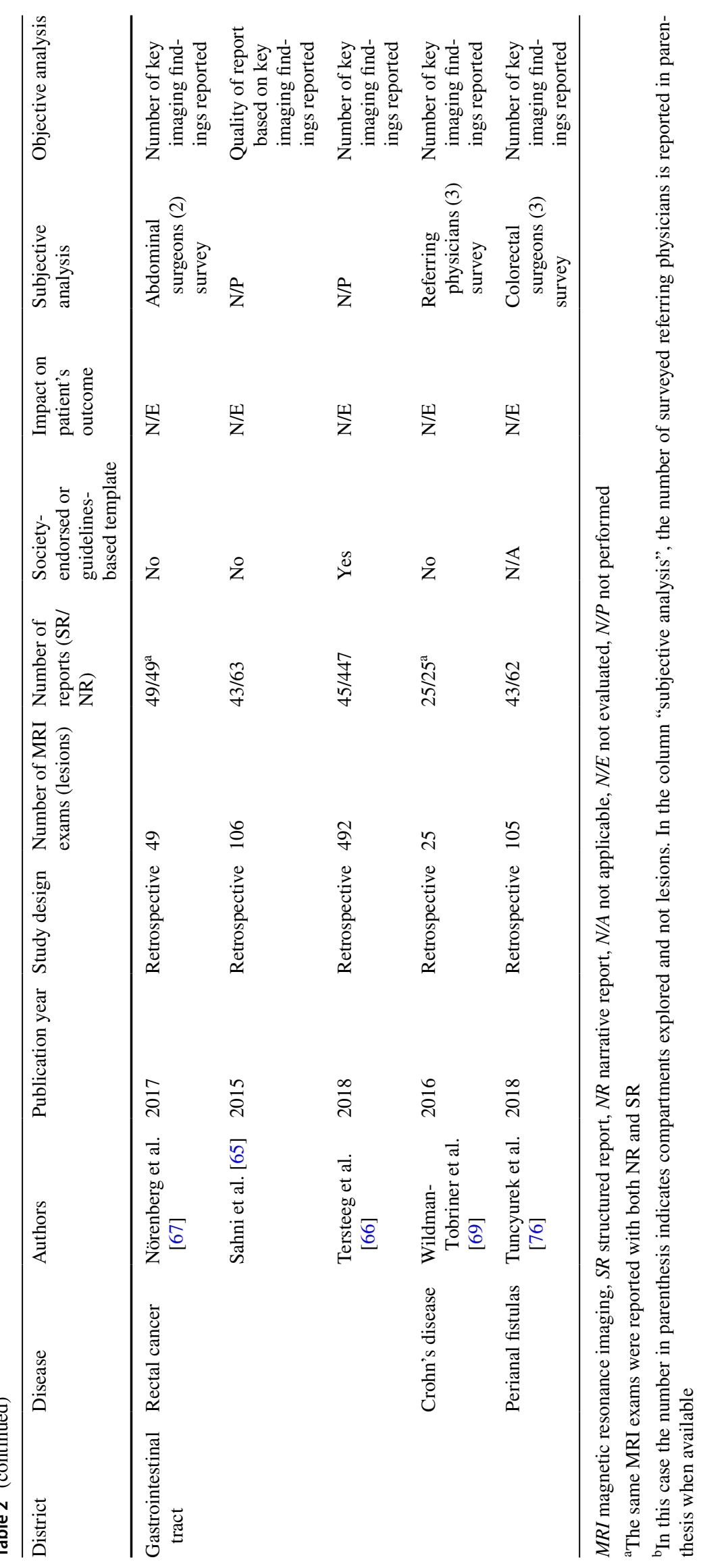


contrast-enhanced MRI and the Liver Imaging-Reporting and Data System (LI-RADS) guidelines have been reported to be highly reliable [12-14]. Indeed, they offer recommendations regarding acquisition technique, key imaging findings and provide both a standardized lexicon and a structured reporting template for HCC diagnosis in high-risk patients [15].

MRI structured reporting for HCC diagnosis might offer added value in terms of reproducibility, with evidence of a standardized reporting system predating the release of LIRADS that showed an intraclass correlation coefficient of 0.80 among 6 radiologists evaluating 100 liver MRI exams [16]. More recently, in a retrospective comparison of a LIRADS based SR with NR, Flusberg et al. found that final LI-RADS category was more frequently included in the first (98\% vs $18 \%, p<0.001$ ), independently of formal LI-RADS training [17]. Similarly, key imaging findings such as arterial phase hyperenhancement and wash out were found in $98 \%$ of SR while not mentioned in $19 \%$ and $26 \%$ of NR, respectively. However, the study does not provide evidence regarding the impact of SR implementation on patient management and outcome.

\section{Renal masses}

Renal masses are commonly detected as incidental findings on imaging examinations and may prove hard to characterize [18]. Therefore, contrast-enhanced MRI protocols have been designed to establish the most appropriate management strategy for indeterminate renal masses [19-21]. Nevertheless, despite an increase in their incidence and prevalence, content of radiological reports was inconsistent and lacking standardization. Thus, in 2015 a dedicated panel convened by the Society of Abdominal Radiology began working on an evidence-based SR template for renal masses. First, they surveyed both radiologists and urologists to define the key imaging findings to be included in a SR [22]. While the inter-specialty consensus was overall good, the urologists were more likely to prefer the inclusion quantitative data (e.g., nephrometry score or the mass relationship to the renal polar lines) and expressed a preference for clinical management recommendations to not be included in radiology reports $[22,23]$. These findings corroborate the need for multidisciplinary engagement to maximize the benefits of SR in terms of communication quality and clinical decision-making. Then, a multicenter, retrospective analysis of radiological reports for indeterminate renal masses was performed and confirmed that some key imaging findings were inconsistently included (such as presence or absence of macroscopic fat -included in 34/236 reports-, size comparisons -included in 111/140 reports- and use of the Bosniak classification for cystic masses -included in 19/35 reports-) with variations across radiologists and practice settings [24].
Finally, evidence-based structured 'core' and 'optional' templates for reporting indeterminate renal masses were realized using the Delphi technique [25]. Unfortunately, studies formally comparing these SR to conventional NR have not yet been performed. However, given the solid methodological ground on which they are based, with their adoption both improved compliance with reporting key imaging findings and increased referring practitioner satisfaction can be expected.

\section{Lower abdomen}

\section{Prostate cancer}

Since the first version of the Prostate Imaging-Reporting and Data System (PI-RADS) guidelines was released, MRI has become the cornerstone for accurate prostate cancer detection [26]. Notably, the use of standardized reporting for prostate MRI had already been investigated by Quentin and colleagues as a feasible tool to guide MRI-targeted rebiopsy [27]. More recently, Wetterauer et al. retrospectively compared $50 \mathrm{NRs}$ and 50 SRs submitting them to the subjective evaluation of 4 experienced urologists who showed an overall higher satisfaction with structured reporting (mean score on a scale from 1 to 5 equal to 4.5 for SR vs. 2.3 for $\mathrm{NR} ; p<0.01)$ and declared a significantly inferior need for radiologists' re-consultation with SR (19\% for SR vs $85 \%$ for $\mathrm{NR} ; p<0.01)$ [28]. Furthermore, the urologists were asked to plot the tumor on a prostate diagram using MRI reports information; when using SR, they showed a higher agreement with a radiologist reviewing the MRI scans to plot the same lesions compared to when using NR. However, all NRs in the study were written in 2011 (before PI-RADS implementation) while SRs between 2015 and 2016 (after PI-RADS version 2 was published). Therefore, the results might be due to the increased experience of reporting radiologists and PI-RADS guidelines adoption rather than to the SR itself. With a quality improvement initiative, Magnetta et al. identified report items deemed essential by local referring urologists (e.g., PI-RADS assessment category, findings listed by lesion, prostate-specific antigen density value and low word count) to design and implement a tailored SR template into their dictation software [29]. This intervention lead to an increase in objective quality (measured in terms of essential items reported) and referring urologist satisfaction, who were significantly less incline to contact radiologists for explanation when provided a SR. However, word count did not drop moving from NR to SR and since the same urologists that selected the essential reporting items subsequently performed the subjective quality analysis, the latter could have been partly biased. Moving from a hybrid NR/SR to a full SR based on PI-RADS lexicon and rules, Shaish and 
colleagues observed a significant improvement of adherence to PI-RADS score rules $(88.4 \%$ versus $32.9 \%, p<0.001$ ) [30]. Nevertheless, a survey of specialty societies found that while $54 \%$ of the urologists prefer structured reporting for prostate MRI, 53\% of the radiologists still indicated a preference towards hybrid NR/SR solutions [31]. Additionally, the preference expressed by radiologists to include clinical management recommendations in their reports is not shared by the urologists, indicating that further interdisciplinary efforts are needed to reach prostate MRI standardized reporting optimization. Finally, beyond prostate cancer detection, prostate MRI standardized reporting might prove useful for local staging as well and scoring system for extraprostatic extension of disease have been proposed [32, 33].

\section{Bladder cancer}

Bladder cancer is associated to high morbidity and mortality, with muscle-invasiveness being a key negative prognostic factor [34]. MRI has been proposed as a feasible diagnostic tool for pre-treatment local staging of the disease and in 2018 the Vesical Imaging-Reporting and Data System (VI-RADS) was released, providing standardized and structured reporting criteria to suggest the likelihood of detrusor muscle invasion [35]. These consensus-based guidelines introduced a five-point probability score derived from T2-weighted, diffusion-weighted and dynamic contrast-enhanced sequence features, and have been endorsed by the Japanese Society of Abdominal Radiology, European Association of Urology, and European Society of Urological Imaging. The validity of VI-RADS has been assessed, with interobserver agreement ranging from good to excellent and an area under the curve for muscle invasion identification as high as 0.94 [36, 37]. Very recently, a meta-analysis performed by Cheng Luo et al. including six studies with 1064 patients confirmed the high diagnostic accuracy of VI-RADS [38]. Furthermore, in a prospective study the VI-RADS score was also found to be a feasible tool to predict the need of repeated transurethral resection of bladder tumor, which is recommended in most high-risk bladder cancer patients not presenting muscle invasion [39]. However, whether the structured format of the MRI report significantly contributes to the success of VI-RADS is still to be verified, as studies formally comparing the use of VI-RADS with SR and NR have not been performed yet.

\section{Gynecological malignancies: ovarian masses and endometrial cancer}

MRI serves as a valuable problem-solving tool for ovarian masses undetermined at ultrasound [40, 41]. In 2013, a standardized reporting system for ovarian mass characterization on MRI was proposed [42]. The validity of this five-category ADNEX MR SCORING system was subsequently verified by independent study groups also confirming that it might lead to the standardization of MRI reporting for ovarian masses [43-45]. Very recently, the MRI working group for the Ovarian-Adnexal Imaging-Reporting and Data System (O-RADS) confirmed with a prospective multicenter study the performance of the ADNEX MR SCORING system (now called the O-RADS MRI score), that represents the current standard in the field [46]. The reproducibility analyses are particularly encouraging, indicating substantial interrater agreement between experienced and junior readers. Therefore, while studies evaluating the role of O-RADS MRI score structured reporting are not yet available, it is fair to assume that the adoption of SR based on the scoring system could be beneficial.

Regarding endometrial cancer, MRI is the imaging modality of choice to perform preoperative disease assessment [47]. In this setting, the European Society of Urogenital Radiology recently released an updated version of their guidelines for endometrial cancer staging with MRI [48]. The working group unanimously agreed on the need for a SR to increase reporting quality and facilitate interdisciplinary communication. A SR template as well as a short mnemonic to summarize the key imaging findings are provided in the guidelines. In a retrospective study, the same radiologist reviewed local staging MRI scans of 41 patients affected by endometrial cancer reporting the images with NR first and using a SR template after 8 weeks and the two reporting strategies were compared [49]. Regarding the objective analysis, the mean number of key features reported was not statistically different between SR and NR $(p=0.055)$. However, the radiologist worked significantly faster when working with the SR $(727.22 \pm 38.42 \mathrm{~s}$ vs. $616.44 \pm 60.00 \mathrm{~s}$, $p=0.037$ ). Finally, it is worth to mention that a SR template for endometrial cancer staging proposed by the Society of Abdominal Radiology and endorsed by the Template Advisory Library Panel can be found at radreport.org.

\section{Benign gynecological disorders}

There is a plethora of non-oncologic gynecological diseases in which MRI plays an important diagnostic role, and many have worked on MRI structured reporting in this area. In a review, expert radiologists proposed a SR for MRI in patients with infertility [50], while two independent panel of experts defined SR templates for standardizing MRI reporting of chronic pelvic pain [51] and female genital tract congenital anomalies [52] using evidence-based approaches and consensus strategies. Regarding endometriosis, several templates for MRI SR have been proposed in the recent literature [53-55]. In particular, the guidelines released by the Female Pelvic Imaging working group of the European Society of Urogenital Radiology include a dedicated "reporting 
criteria" section for MRI of endometriosis in which criteria for the diagnosis of endometrial cysts and adhesions as well as the possible locations of deep pelvic endometriosis are defined [55]. However, in a per-location retrospective analysis of 295 compartments (59 patients with surgically confirmed diagnosis), a recent investigation concluded that reader experience may have a greater impact on MRI diagnostic accuracy for deep pelvic endometriosis than reporting style [56].

In the field of benign tumors, the possible role of SR for uterine fibroids MRI has been explored. After the introduction of SR in their department, Franconeri and colleagues retrospectively reported that key features were less consistently included in NR compared to SR $(7.3 \pm 2.5$ vs $1.2 \pm 1.5$ missing key feature, $p<0.0001$ ) [57]. Accordingly, referring practitioners qualitatively described SR as more helpful for treatment planning and easier to understand. Nevertheless, the SR was realized with a multidisciplinary approach which might have affected the qualitative analysis.

\section{Gastrointestinal tract}

\section{Rectal cancer}

While the prevalence of rectal cancer is rising in developed countries, mortality rate has been declining at least partly due to the added value of MRI in pre- and post-treatment assessment of the disease [58]. Indeed, MRI allows to identify patients that should receive neoadjuvant chemotherapy, aids in surgical approaches customization and can be used for treatment response assessment [59]. With specific regard to rectal cancer MRI staging, a systematic approach to standardize the reporting process was first described in 2008 [60] and shortly after the same working group proposed a SR template including all relevant key imaging findings [61]. Interestingly, in 2014 a synoptic MRI report was successfully developed, and pilot tested in Ontario (Canada), with a 37\% adoption rate across the province and leading to a 39\% improvement in MRI reports completeness [62]. More recently, the results of scientific societies consensus meetings focused on rectal cancer MRI staging have been released, defining essential items and SR templates for structured reporting $[63,64]$. There are some publications suggesting that the use of SR over NR could be beneficial in this field. Sahni et al. found that implementing a SR template and its voluntary use in a radiology department increased the quality of MRI reports for rectal cancer staging, with the percentage of reports including all key imaging features rising from 0 with NR to $41 \%$ with SR [65]. However, $30 \%$ of all reports produced after the intervention were still unsatisfactory, half of which belonging to the group (20\%) that continued to use NR. In the Netherlands, a significant increase in the number of key imaging features reported was observed after the introduction of new guidelines and SR templates for rectal cancer MRI staging (from a median value of $4 / 10$ to $9 / 10$ items, $p<0.001$ ) [66]. Noremberg and colleagues evaluated the impact of MRI SR compared to NR in clinical decision-making (surgery vs neoadjuvant radiochemotherapy) for patients with rectal cancer and found that SR allowed a definite choice in $96 \%$ of cases vs $60 \%$ of NR [67]. Furthermore, SR were considered adequate for surgical planning in $94 \%$ of cases while only $38 \%$ of NR were judged so by surgeons. However, due to the retrospective design of the study and the lack of a correctness evaluation of reported finding, final conclusions on the clinical impact of SR for rectal cancer MRI staging could not be drawn. Nevertheless, structured reporting appears to be a feasible tool to ensure essential information influencing rectal cancer management are conveyed to referring physicians.

\section{Crohn's disease}

Crohn's disease is a chronic inflammatory bowel disorder which typically involves multiple segments of the gastrointestinal tract, at different time points and with varying degrees of severity [68]. While crucial information can be obtained from clinical and endoscopic evaluation, crosssectional imaging modalities such as magnetic resonance enterography (MRE), performed with administration contrast mediums both per os and per venam, is often necessary to assess disease status and guide treatment [69]. Recently, The Society of Abdominal Radiology (SAR) Crohn DiseaseFocused Panel together with the American Gastroenterological Association (AGA) defined consensus recommendations regarding the use, interpretation and reporting of MRE [70]. In this work, a SR template based on a previous experience is proposed [71]. Rees et al. assessed the inter-reader agreement when using the SAR-AGA recommendations and found a moderate to substantial agreement among five radiologists using structured MRE templates [72]. Since the better results were found for conditions most likely to require intervention (e.g., structuring disease), the authors speculate that SR might influence Crohn's disease management. Before the SAR-AGA guidelines were released, Wildmann and colleagues compared MRE SR and NR in a population of pediatric patients with Crohn's disease [69]. They concluded that radiologists objectively detailed nearly two times more key features with structured reporting (a mean of $7.7 \pm 2.5$ key features for NR compared to $14.0 \pm 0.8$ for SR, $p<0.001)$. Additionally, the subjective comparative analysis of SR and NR performed by referring physicians revealed a preference towards SR due to higher clarity. However, these retrospective studies included a small number of MRE which were reviewed for the purposes of the analyses and therefore the results might not apply to daily reporting workflow. 


\section{Perianal fistulas}

Perianal fistulas (or fistula-in-ano, FIA) are inflammatory disorders of the anus and perianal soft tissue that can be a cause of substantial morbidity, often requiring surgical intervention [73]. Patients are frequently referred to MRI for the assessment of FIA extent, to verify presence of complications and to confirm the diagnosis in challenging cases. Recently, SR templates for MRI of FIA have been published, sharing similar key imaging features (e.g., FIA type, extension and accessory tracts, presence of abscess) and highlighting the importance of clock-face annotation to indicate position $[74,75]$. In both cases, the authors emphasized that SR templates could optimize patient management through clear and complete communication of key imaging findings while reducing reporting time and making MRI studies easier to compare. Performing a retrospective comparison, Tuncyurek et al. confirmed that a disease-specific SR is superior to conventional reporting for FIA MRI [76]. Specifically, an objective analysis found that in NR more key features were absent than in SR (respectively $6.3 \pm 1.8$ vs $0.3 \pm 0.9, p \leq 0.001)$. In the subjective surgeons' judgment, SR was also deemed to be clearer compared to NR. Of note, the authors highlight the importance of education on top of report standardization since radiologists that kept using the NR after SR introduction began including more key imaging features too.

\section{Discussion}

Physicians rely on a written interpretation of MRI findings to assist them in patient management while patients demand clearer, more accessible radiology reports [2]. They are the most important element on which our work is assessed, and NR have been often criticized as a vestige of the past and at risk of being inconsistent and therefore unreliable [77]. While possibly offering a solution to this problem, the implementation of SRs is a complex and debated issue [78]. There are recognized limitations of SR, such as the risk of excessive simplification and template layout rigidity as well as poor user compliance [79]. Nevertheless, as described previously significant efforts have been made by scientific societies and panels of experts towards the definition of common SR templates for abdominal MRI in various settings. Their implementation in radiology departments with high adoption rates might not be overly complicated and tailoring on departmental specific characteristics and in accordance with local referring physicians is possible and should be considered [80]. Herts et al. propose to realize a system-specific report and then disease-specific report templates by expanding on the first [10]. Such an approach could allow SR to appear less rigid and be more easily embraced. However, this might still prove challenging and SR templates might not be able to meet every reporting need as they appear best suited for specific conditions rather than complex patients [78]. Additionally, many among the SR templates presented in this review have only been released very recently. Another issue that emerges from this review of the literature is the lack of studies proving a significant benefit for patient outcome associated to SR. Currently available data from formal comparisons between NR and SR are based on subjective (perceived quality questionnaires) and objective (key imaging findings reported) evaluations performed retrospectively, which do not provide the desirable level of evidence to confirm the urgency for a widespread adoption of structured reporting. It is possible that the same advantages of SR use could be obtained by proper education of subspecialty radiologists and promoting the use of standardized lexicon and validated scoring systems without the need for actual structuring of reports, therefore defending the autonomy of radiologists [81]. It could also be argued that multidisciplinary meetings and radiologist consultations might be a different feasible solution to increase the value and impact of our work [82]. If prospective studies designed to clarify these issues will not be performed, there is a risk that the debate on reporting strategies will not be settled, limiting SR implementation. Finally, there are diseases in which the possible advantage of MRI SR remains largely unexplored (e.g., pancreatic cystic lesions, adrenal masses, biliary duct pathology, outlet obstruction).

In conclusion, the assessment of certain abdominal disorders by means of MRI might benefit from the adoption of SR over NR in terms of higher reporting quality and referring physician's satisfaction. Current evidence suggests that SR tends to be more comprehensive, including a higher number of key imaging findings compared to NR. A multitude of templates is already available, but further and stronger evidence confirming their value is needed, especially regarding patient's outcome improvement.

Author contributions AS conceptualized the review. AS, FB, RC and VR performed the literature search and contributed to drafting the manuscript. SM, AB and PPM oversaw the work and edited the manuscript. All authors read and approved the final manuscript.

Funding Open access funding provided by Università degli Studi di Napoli Federico II within the CRUI-CARE Agreement.

\section{Compliance with ethical standards}

Conflict of interest The authors declare that they have no competing interests. 
Open Access This article is licensed under a Creative Commons Attribution 4.0 International License, which permits use, sharing, adaptation, distribution and reproduction in any medium or format, as long as you give appropriate credit to the original author(s) and the source, provide a link to the Creative Commons licence, and indicate if changes were made. The images or other third party material in this article are included in the article's Creative Commons licence, unless indicated otherwise in a credit line to the material. If material is not included in the article's Creative Commons licence and your intended use is not permitted by statutory regulation or exceeds the permitted use, you will need to obtain permission directly from the copyright holder. To view a copy of this licence, visit http://creativecommons.org/licenses/by/4.0/.

\section{References}

1. Panicek DM, Hricak H (2019) Recommendations for the Initial Cancer Staging MRI Report. J Magn Reson Imaging 49:12091211. https://doi.org/10.1002/jmri.26528

2. Alper DP, Shinagare AB, Hashemi SR, et al (2020) Effect of a Report Template-Enabled Quality Improvement Initiative on Use of Preferred Phrases for Communicating Normal Findings in Structured Abdominal CT and MRI Reports. Am J Roentgenol 214:835-842. https://doi.org/10.2214/AJR.19.21735

3. (2018) ESR paper on structured reporting in radiology. Insights Imaging 9:1-7. https://doi.org/10.1007/s13244-017-0588-8

4. Ganeshan D, Duong P-AT, Probyn L, et al (2018) Structured Reporting in Radiology. Acad Radiol 25:66-73. https://doi. org/10.1016/j.acra.2017.08.005

5. Langlotz C (2015) The Radiology Report: A Guide to Thoughtful Communication for Radiologists and Other Medical Professionals.

6. Sala E, Freeman S (2018) Structured reporting of pelvic MRI leads to better treatment planning of uterine leiomyomas. Eur Radiol 28:3007-3008. https://doi.org/10.1007/s00330-018-5417-z

7. Hawkins CM, Hall S, Zhang B, Towbin AJ (2014) Creation and Implementation of Department-Wide Structured Reports: An Analysis of the Impact on Error Rate in Radiology Reports. J Digit Imaging 27:581-587. https://doi.org/10.1007/s10278-014-9699-7

8. Weiss DL, Langlotz CP (2008) Structured Reporting: Patient Care Enhancement or Productivity Nightmare? Radiology 249:739-747. https://doi.org/10.1148/radiol.2493080988

9. Nobel JM, Kok EM, Robben SGF (2020) Redefining the structure of structured reporting in radiology. Insights Imaging 11:10. https://doi.org/10.1186/s13244-019-0831-6

10. Herts BR, Gandhi NS, Schneider E, et al (2019) How We Do It: Creating Consistent Structure and Content in Abdominal Radiology Report Templates. Am J Roentgenol 212:490-496. https ://doi.org/10.2214/AJR.18.20368

11. Galle PR, Forner A, Llovet JM, et al (2018) EASL Clinical Practice Guidelines: Management of hepatocellular carcinoma. J Hepatol 69:182-236. https://doi.org/10.1016/j.jhep.2018.03.019

12. Shah A, Tang A, Santillan C, Sirlin C (2016) Cirrhotic liver: What's that nodule? The LI-RADS approach. J Magn Reson Imaging 43:281-294. https://doi.org/10.1002/jmri.24937

13. Lee S, Kim M-J, Kim S, et al (2020) Retrospective comparison of EASL 2018 and LI-RADS 2018 for the noninvasive diagnosis of hepatocellular carcinoma using magnetic resonance imaging. Hepatol Int 14:70-79. https://doi.org/10.1007/s1207 2-019-10002-3

14. Imbriaco M, De Luca S, Coppola M, et al (2017) Diagnostic Accuracy of Gd-EOB-DTPA for Detection Hepatocellular Carcinoma (HCC): A Comparative Study with Dynamic Contrast Enhanced Magnetic Resonance Imaging (MRI) and Dynamic
Contrast Enhanced Computed Tomography (CT). Polish J Radiol 82:50-57. https://doi.org/10.12659/PJR.899239

15. Reporting and Data Systems, America College of Radiology. www.acr.org/Clinical-Resources/Reporting-and-Data-Systems/. Accessed 26 Aug 2020

16. Petruzzi N, Mitchell D, Guglielmo F, et al (2013) Hepatocellular Carcinoma Likelihood on MRI Exams. Acad Radiol 20:694698. https://doi.org/10.1016/j.acra.2013.01.016

17. Flusberg M, Ganeles J, Ekinci T, et al (2017) Impact of a Structured Report Template on the Quality of CT and MRI Reports for Hepatocellular Carcinoma Diagnosis. J Am Coll Radiol 14:1206-1211. https://doi.org/10.1016/j.jacr.2017.02.050

18. Volpe A, Panzarella T, Rendon RA, et al (2004) The natural history of incidentally detected small renal masses. Cancer 100:738-745. https://doi.org/10.1002/cncr.20025

19. Lopes Vendrami C, Parada Villavicencio C, DeJulio TJ, et al (2017) Differentiation of Solid Renal Tumors with Multiparametric MR Imaging. RadioGraphics 37:2026-2042. https://doi. org/10.1148/rg.2017170039

20. Sasaguri K, Takahashi N (2018) CT and MR imaging for solid renal mass characterization. Eur J Radiol 99:40-54. https://doi. org/10.1016/j.ejrad.2017.12.008

21. Baldari D, Capece S, Mainenti PP, et al (2015) Comparison between computed tomography multislice and high-field magnetic resonance in the diagnostic evaluation of patients with renal masses. Quant Imaging Med Surg 5:691-9. https://doi. org/10.3978/j.issn.2223-4292.2015.07.03

22. Davenport MS, Hu EM, Smith AD, et al (2017) Reporting standards for the imaging-based diagnosis of renal masses on CT and MRI: a national survey of academic abdominal radiologists and urologists. Abdom Radiol 42:1229-1240. https://doi. org/10.1007/s00261-016-0962-x

23. Alsaikhan N, Alshehri W, Cassidy F, et al (2019) Renal tumor structured reporting including nephrometry score and beyond: what the urologist and interventional radiologist need to know. Abdom Radiol 44:190-200. https://doi.org/10.1007/s0026 1-018-1691-0

24. Hu EM, Zhang A, Silverman SG, et al (2018) Multi-institutional analysis of CT and MRI reports evaluating indeterminate renal masses: comparison to a national survey investigating desired report elements. Abdom Radiol 43:3493-3502. https://doi. org/10.1007/s00261-018-1609-x

25. Davenport MS, Hu EM, Zhang A, et al (2019) Standardized report template for indeterminate renal masses at CT and MRI: a collaborative product of the SAR Disease-Focused Panel on Renal Cell Carcinoma. Abdom Radiol 44:1423-1429. https:// doi.org/10.1007/s00261-018-1851-2

26. Kasivisvanathan V, Rannikko AS, Borghi M, et al (2018) MRITargeted or Standard Biopsy for Prostate-Cancer Diagnosis. N Engl J Med 378:1767-1777. https://doi.org/10.1056/NEJMo a1801993

27. Quentin M, Blondin D, Klasen J, et al (2012) Evaluation of a Structured Report of Functional Prostate Magnetic Resonance Imaging in Patients with Suspicion for Prostate Cancer or under Active Surveillance. Urol Int 89:25-29. https://doi. org/10.1159/000338808

28. Wetterauer C, Winkel DJ, Federer-Gsponer JR, et al (2019) Structured reporting of prostate magnetic resonance imaging has the potential to improve interdisciplinary communication. PLoS One 14:e0212444. https://doi.org/10.1371/journ al.pone. 0212444

29. Magnetta MJ, Donovan AL, Jacobs BL, et al (2018) EvidenceBased Reporting: A Method to Optimize Prostate MRI Communications With Referring Physicians. Am J Roentgenol 210:108112. https://doi.org/10.2214/AJR.17.18260 
30. Shaish H, Feltus W, Steinman J, et al (2018) Impact of a Structured Reporting Template on Adherence to Prostate Imaging Reporting and Data System Version 2 and on the Diagnostic Performance of Prostate MRI for Clinically Significant Prostate Cancer. J Am Coll Radiol 15:749-754. https://doi.org/10.1016/j. jacr.2018.01.034

31. Spilseth B, Ghai S, Patel NU, et al (2018) A Comparison of Radiologists' and Urologists' Opinions Regarding Prostate MRI Reporting: Results From a Survey of Specialty Societies. Am J Roentgenol 210:101-107. https://doi.org/10.2214/AJR.17.18241

32. Wibmer A, Vargas HA, Sosa R, et al (2014) Value of a Standardized Lexicon for Reporting Levels of Diagnostic Certainty in Prostate MRI. Am J Roentgenol 203:W651-W657. https://doi. org/10.2214/AJR.14.12654

33. Mehralivand S, Shih JH, Harmon S, et al (2019) A Grading System for the Assessment of Risk of Extraprostatic Extension of Prostate Cancer at Multiparametric MRI. Radiology 290:709-719. https://doi.org/10.1148/radiol.2018181278

34. Kamat AM, Hahn NM, Efstathiou JA, et al (2016) Bladder cancer. Lancet 388:2796-2810. https://doi.org/10.1016/S0140 $-6736(16) 30512-8$

35. Panebianco V, Narumi Y, Altun E, et al (2018) Multiparametric Magnetic Resonance Imaging for Bladder Cancer: Development of VI-RADS (Vesical Imaging-Reporting And Data System). Eur Urol 74:294-306. https://doi.org/10.1016/j.eururo.2018.04.029

36. Barchetti G, Simone G, Ceravolo I, et al (2019) Multiparametric MRI of the bladder: inter-observer agreement and accuracy with the Vesical Imaging-Reporting and Data System (VI-RADS) at a single reference center. Eur Radiol 29:5498-5506. https://doi. org/10.1007/s00330-019-06117-8

37. Wang H, Luo C, Zhang F, et al (2019) Multiparametric MRI for Bladder Cancer: Validation of VI-RADS for the Detection of Detrusor Muscle Invasion. Radiology 291:668-674. https://doi. org/10.1148/radiol.2019182506

38. Luo C, Huang B, Wu Y, et al (2020) Use of Vesical ImagingReporting and Data System (VI-RADS) for detecting the muscle invasion of bladder cancer: a diagnostic meta-analysis. Eur Radiol 30:4606-4614. https://doi.org/10.1007/s00330-020-06802-z

39. Del Giudice F, Barchetti G, De Berardinis E, et al (2020) Prospective Assessment of Vesical Imaging Reporting and Data System (VI-RADS) and Its Clinical Impact on the Management of Highrisk Non-muscle-invasive Bladder Cancer Patients Candidate for Repeated Transurethral Resection. Eur Urol 77:101-109. https:// doi.org/10.1016/j.eururo.2019.09.029

40. Glanc P, Benacerraf B, Bourne T, et al (2017) First International Consensus Report on Adnexal Masses: Management Recommendations. J Ultrasound Med 36:849-863. https://doi.org/10.1002/ jum.14197

41. Forstner R, Thomassin-Naggara I, Cunha TM, et al (2017) ESUR recommendations for MR imaging of the sonographically indeterminate adnexal mass: an update. Eur Radiol 27:2248-2257. https ://doi.org/10.1007/s00330-016-4600-3

42. Thomassin-Naggara I, Aubert E, Rockall A, et al (2013) Adnexal Masses: Development and Preliminary Validation of an MR Imaging Scoring System. Radiology 267:432-443. https://doi. org/10.1148/radiol.13121161

43. Ruiz M, Labauge P, Louboutin A, et al (2016) External validation of the MR imaging scoring system for the management of adnexal masses. Eur J Obstet Gynecol Reprod Biol 205:115-119. https:// doi.org/10.1016/j.ejogrb.2016.07.493

44. Sasaguri K, Yamaguchi K, Nakazono T, et al (2019) External validation of ADNEX MR SCORING system: a single-centre retrospective study. Clin Radiol 74:131-139. https://doi.org/10.1016/j. crad.2018.10.014

45. Pereira PN, Sarian LO, Yoshida A, et al (2018) Accuracy of the ADNEX MR scoring system based on a simplified MRI protocol for the assessment of adnexal masses. Diagnostic Interv Radiol. https://doi.org/10.5152/dir.2018.17378

46. Thomassin-Naggara I, Poncelet E, Jalaguier-Coudray A, et al (2020) Ovarian-Adnexal Reporting Data System Magnetic Resonance Imaging (O-RADS MRI) Score for Risk Stratification of Sonographically Indeterminate Adnexal Masses. JAMA Netw Open 3:e1919896. https://doi.org/10.1001/jamanetworkopen .2019 .19896

47. Luomaranta A, Leminen A, Loukovaara M (2015) Magnetic Resonance Imaging in the Assessment of High-Risk Features of Endometrial Carcinoma. Int J Gynecol Cancer 25:837-842. https ://doi.org/10.1097/IGC.0000000000000194

48. Nougaret S, Horta M, Sala E, et al (2019) Endometrial Cancer MRI staging: Updated Guidelines of the European Society of Urogenital Radiology. Eur Radiol 29:792-805. https://doi. org/10.1007/s00330-018-5515-y

49. Liu Y, Feng Z, Qin S, et al (2019) Structured reports of pelvic magnetic resonance imaging in primary endometrial cancer: Potential benefits for clinical decision-making. PLoS One 14:e0213928. https://doi.org/10.1371/journal.pone.0213928

50. Montoliu-Fornas G, Martí-Bonmatí L (2016) Magnetic resonance imaging structured reporting in infertility. Fertil Steril 105:14211431. https://doi.org/10.1016/j.fertnstert.2016.04.005

51. Bharwani N, Tirlapur SA, Balogun M, et al (2016) MRI reporting standard for chronic pelvic pain: consensus development. $\mathrm{Br}$ J Radiol 89:20140615. https://doi.org/10.1259/bjr.20140615

52. Maciel C, Bharwani N, Kubik-Huch RA, et al (2020) MRI of female genital tract congenital anomalies: European Society of Urogenital Radiology (ESUR) guidelines. Eur Radiol. https://doi. org/10.1007/s00330-020-06750-8

53. Feldman MK, VanBuren WM, Barnard H, et al (2020) Systematic interpretation and structured reporting for pelvic magnetic resonance imaging studies in patients with endometriosis: value added for improved patient care. Abdom Radiol 45:1608-1622. https:// doi.org/10.1007/s00261-019-02182-1

54. Mattos LA, Goncalves MO, Andres MP, et al (2019) Structured Ultrasound and Magnetic Resonance Imaging Reports for Patients with Suspected Endometriosis: Guide for Imagers and Clinicians. J Minim Invasive Gynecol 26:1016-1025. https://doi. org/10.1016/j.jmig.2019.02.017

55. Bazot M, Bharwani N, Huchon C, et al (2017) European society of urogenital radiology (ESUR) guidelines: MR imaging of pelvic endometriosis. Eur Radiol 27:2765-2775. https://doi.org/10.1007/ s00330-016-4673-z

56. Jaramillo-Cardoso A, Shenoy-Bhangle A, Garces-Descovich A, et al (2020) Pelvic MRI in the diagnosis and staging of pelvic endometriosis: added value of structured reporting and expertise. Abdom Radiol 45:1623-1636. https://doi.org/10.1007/s0026 1-019-02199-6

57. Franconeri A, Fang J, Carney B, et al (2018) Structured vs narrative reporting of pelvic MRI for fibroids: clarity and impact on treatment planning. Eur Radiol 28:3009-3017. https://doi. org/10.1007/s00330-017-5161-9

58. Jhaveri KS, Hosseini-Nik H (2015) MRI of Rectal Cancer: An Overview and Update on Recent Advances. Am J Roentgenol 205:W42-W55. https://doi.org/10.2214/AJR.14.14201

59. Horvat N, Carlos Tavares Rocha C, Clemente Oliveira B, et al (2019) MRI of Rectal Cancer: Tumor Staging, Imaging Techniques, and Management. RadioGraphics 39:367-387. https://doi. org/10.1148/rg.2019180114

60. Taylor FGM, Swift RI, Blomqvist L, Brown G (2008) A Systematic Approach to the Interpretation of Preoperative Staging MRI for Rectal Cancer. Am J Roentgenol 191:1827-1835. https://doi. org/10.2214/AJR.08.1004 
61. Taylor F (2010) Proforma-based reporting in rectal cancer. Cancer Imaging 10:S142-S150. https://doi. org/10.1102/1470-7330.2010.9092

62. Kennedy ED, Milot L, Fruitman M, et al (2014) Development and Implementation of a Synoptic MRI Report for Preoperative Staging of Rectal Cancer on a Population-Based Level. Dis Colon Rectum 57:700-708. https://doi.org/10.1097/DCR.0000000000 000123

63. (2017) Essential Items for Structured Reporting of Rectal Cancer MRI: 2016 Consensus Recommendation from the Korean Society of Abdominal Radiology. Korean J Radiol 18:132. https://doi. org/10.3348/kjr.2017.18.1.132

64. Beets-Tan RGH, Lambregts DMJ, Maas M, et al (2018) Magnetic resonance imaging for clinical management of rectal cancer: Updated recommendations from the 2016 European Society of Gastrointestinal and Abdominal Radiology (ESGAR) consensus meeting. Eur Radiol 28:1465-1475. https://doi.org/10.1007/s0033 0-017-5026-2

65. Sahni VA, Silveira PC, Sainani NI, Khorasani R (2015) Impact of a Structured Report Template on the Quality of MRI Reports for Rectal Cancer Staging. Am J Roentgenol 205:584-588. https ://doi.org/10.2214/AJR.14.14053

66. Tersteeg JJC, Gobardhan PD, Crolla RMPH, et al (2018) Improving the Quality of MRI Reports of Preoperative Patients With Rectal Cancer: Effect of National Guidelines and Structured Reporting. Am J Roentgenol 210:1240-1244. https://doi.org/10.2214/ AJR.17.19054

67. Nörenberg D, Sommer WH, Thasler W, et al (2017) Structured Reporting of Rectal Magnetic Resonance Imaging in Suspected Primary Rectal Cancer. Invest Radiol 52:232-239. https://doi. org/10.1097/RLI.0000000000000336

68. Torres J, Mehandru S, Colombel J-F, Peyrin-Biroulet L (2017) Crohn's disease. Lancet 389:1741-1755. https://doi.org/10.1016/ S0140-6736(16)31711-1

69. Wildman-Tobriner B, Allen BC, Davis JT, et al (2017) Structured Reporting of Magnetic Resonance Enterography for Pediatric Crohn's Disease: Effect on Key Feature Reporting and Subjective Assessment of Disease by Referring Physicians. Curr Probl Diagn Radiol 46:110-114. https://doi.org/10.1067/j.cprad iol.2016.12.001

70. Bruining DH, Zimmermann EM, Loftus E V., et al (2018) Consensus Recommendations for Evaluation, Interpretation, and Utilization of Computed Tomography and Magnetic Resonance Enterography in Patients With Small Bowel Crohn's Disease. Gastroenterology 154:1172-1194. https://doi.org/10.1053/j.gastr o.2017.11.274

71. Baker ME, Hara AK, Platt JF, et al (2015) CT enterography for Crohn's disease: optimal technique and imaging issues. Abdom Imaging 40:938-952. https://doi.org/10.1007/s00261-015-0357-4
72. Rees MA, Dillman JR, Anton CG, et al (2019) Inter-radiologist agreement using Society of Abdominal Radiology-American Gastroenterological Association (SAR-AGA) consensus nomenclature for reporting CT and MR enterography in children and young adults with small bowel Crohn disease. Abdom Radiol 44:391397. https://doi.org/10.1007/s00261-018-1743-5

73. Tolan DJM (2016) Magnetic Resonance Imaging for Perianal Fistula. Semin Ultrasound, CT MRI 37:313-322. https://doi. org/10.1053/j.sult.2016.04.004

74. Thipphavong S, Costa AF, Ali HA, et al (2019) Structured reporting of MRI for perianal fistula. Abdom Radiol 44:1295-1305. https://doi.org/10.1007/s00261-018-1839-y

75. Ho E, Rickard MJFX, Suen M, et al (2019) Perianal sepsis: surgical perspective and practical MRI reporting for radiologists. Abdom Radiol 44:1744-1755. https://doi.org/10.1007/s0026 1-019-01920-9

76. Tuncyurek O, Garces-Descovich A, Jaramillo-Cardoso A, et al (2019) Structured versus narrative reporting of pelvic MRI in perianal fistulizing disease: impact on clarity, completeness, and surgical planning. Abdom Radiol 44:811-820. https://doi. org/10.1007/s00261-018-1858-8

77. Rao VM, Levin DC (2011) The Value-Added Services of Hospital-Based Radiology Groups. J Am Coll Radiol 8:626-630. https ://doi.org/10.1016/j.jacr.2011.03.007

78. Powell DK, Silberzweig JE (2015) State of Structured Reporting in Radiology, a Survey. Acad Radiol 22:226-233. https://doi. org/10.1016/j.acra.2014.08.014

79. Faggioni L, Coppola F, Ferrari R, et al (2017) Usage of structured reporting in radiological practice: results from an Italian online survey. Eur Radiol 27:1934-1943. https://doi.org/10.1007/s0033 0-016-4553-6

80. Larson DB, Towbin AJ, Pryor RM, Donnelly LF (2013) Improving Consistency in Radiology Reporting through the Use of Department-wide Standardized Structured Reporting. Radiology 267:240-250. https://doi.org/10.1148/radiol.12121502

81. Palmer W, Bancroft L, Bonar F, et al (2020) Glossary of terms for musculoskeletal radiology. Skeletal Radiol 49:1-33. https://doi. org/10.1007/s00256-020-03465-1

82. Pillay B, Wootten AC, Crowe H, et al (2016) The impact of multidisciplinary team meetings on patient assessment, management and outcomes in oncology settings: A systematic review of the literature. Cancer Treat Rev 42:56-72. https://doi.org/10.1016/j. ctrv.2015.11.007

Publisher's Note Springer Nature remains neutral with regard to jurisdictional claims in published maps and institutional affiliations. 